\title{
The GEO-HF project
}

B Willke ${ }^{1}$, P Ajith ${ }^{1}$, B Allen $^{3}$, P Aufmuth ${ }^{1}$, C Aulbert $^{3}$, S Babak $^{3}$, R Balasubramanian ${ }^{4}$, B W Barr ${ }^{3}$, S Berukoff $^{3}$, A Bunkowski ${ }^{1}$, G Cagnoli ${ }^{2}$, C A Cantley ${ }^{2}$, M M Casey ${ }^{2}$, S Chelkowski ${ }^{1}, \mathrm{Y} \mathrm{Chen}^{3}$,

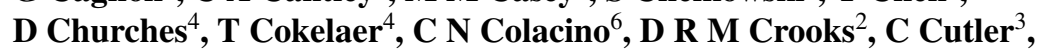
K Danzmann ${ }^{1}$, R J Dupuis ${ }^{2}$, E Elliffe ${ }^{2}$, C Fallnich ${ }^{5}$, A Franzen ${ }^{1}$, A Freise ${ }^{1}$, I Gholami $^{3}$, S Goßler ${ }^{1}$, A Grant ${ }^{2}$, H Grote ${ }^{1}$, S Grunewald ${ }^{3}$, J Harms ${ }^{1}$,

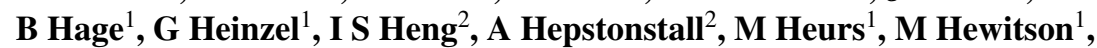
S Hild ${ }^{1}$, J Hough ${ }^{2}$, Y Itoh ${ }^{3}$, G Jones ${ }^{4}$, R Jones ${ }^{2}$, S H Huttner ${ }^{2}$, K Kötter ${ }^{1}$, B Krishnan $^{3}$, P Kwee ${ }^{1}$, H Lück ${ }^{1}$, Luna $^{7}$, B Machenschalk ${ }^{3}$, M Malec ${ }^{1}$, R A Mercer ${ }^{6}$, T Meier ${ }^{1}, \mathbf{C}$ Messenger $^{6}, \mathbf{S}$ Mohanty $^{3}, \mathbf{K}^{3}$ Mossavi $^{1}$, S Mukherjee ${ }^{3}$, P Murray ${ }^{2}$, G P Newton ${ }^{2}$, M A Papa ${ }^{3}$, M Perreur-Lloyd ${ }^{2}$,

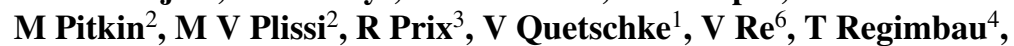
H Rehbein ${ }^{1}$, S Reid ${ }^{2}$, L Ribichini ${ }^{1}$, D I Robertson ${ }^{2}$, N A Robertson ${ }^{2,8}$, C Robinson ${ }^{4}$, J D Romano ${ }^{4}$, S Rowan ${ }^{2}$, A Rüdiger ${ }^{1}$, B S Sathyaprakash ${ }^{4}$, R Schilling ${ }^{1}$, R Schnabel ${ }^{1}$, B F Schutz ${ }^{3,4}$, F Seifert ${ }^{1}$, A M Sintes ${ }^{7}$, J R Smith ${ }^{1}$, P H Sneddon ${ }^{2}$, K A Strain ${ }^{2}$, I Taylor ${ }^{4}$, R Taylor ${ }^{2}$, A Thüring ${ }^{1}$, C Ungarelli ${ }^{6}, \mathbf{H}$ Vahlbruch $^{1}, \mathbf{A}$ Vecchio $^{6}, \mathbf{J ~ V e i t c h ~}^{2}, \mathbf{H}$ Ward $^{2}, \mathbf{U}_{\text {Weiland }}{ }^{1}$, H Welling ${ }^{5}$, L Wen ${ }^{3}$, P Williams ${ }^{3}$, W Winkler ${ }^{1}$, G Woan ${ }^{2}$ and R Zhu ${ }^{3}$

\footnotetext{
${ }^{1}$ Max-Planck-Institut für Gravitationsphysik (Albert-Einstein-Institut) und Universität Hannover, Callinstr 38, D-30167 Hannover, Germany

2 Department of Physics \& Astronomy, University of Glasgow, Glasgow G12 8QQ, UK

${ }^{3}$ Max-Planck-Institut für Gravitationsphysik (Albert-Einstein-Institut), Golm Am Mühlenberg 1, D-14476 Golm, Germany

${ }^{4}$ Department of Physics and Astronomy, Cardiff University, PO Box 913, Cardiff, CF2 3YB, UK

${ }^{5}$ Laser Zentrum Hannover eV, Hollerithallee 8, D-30419 Hannover, Germany

${ }^{6}$ School of Physics and Astronomy, The University of Birmingham, Edgbaston, Birmingham, B15 2TT, UK

${ }^{7}$ Departament de Fisica, Universitat de les Illes Balears, E-07122 Palma de Mallorca, Spain

${ }^{8}$ Edward L Ginzton Laboratory, Stanford University, Stanford, CA 94305-4088, USA
}

Received 31 August 2005, in final form 25 November 2005

Published 28 March 2006

Online at stacks.iop.org/CQG/23/S207

\begin{abstract}
The GEO 600 gravitational wave detector uses advanced technologies including signal recycling and monolithic fused-silica suspensions to achieve a sensitivity close to the kilometre scale LIGO and VIRGO detectors. As soon as the design sensitivity of GEO 600 is reached, the detector will be operated as part of the worldwide network to acquire data of scientific interest. The limited infrastructure at the GEO site does not allow for a major upgrade of the detector. Hence the GEO collaboration decided to improve the sensitivity of the GEO detector by small sequential upgrades some of which will be tested
\end{abstract}


in prototypes first. The development, test and installation of these upgrades are named 'The GEO-HF Project.' This paper describes the upgrades considered in the GEO-HF project as well as their scientific reasons. We will describe the changes in the GEO 600 infrastructure and the prototype work that is planned to support these upgrades. Finally, we will point to some laboratory research that identifies new technologies or optical configurations that might undergo a transition into detector subsystems within the GEO-HF project.

PACS numbers: $04.80 . \mathrm{Nn}$, 07.60.Ly, 95.45.+i, 95.55.Ym

(Some figures in this article are in colour only in the electronic version)

\section{Introduction}

Several laser interferometric gravitational wave detectors worldwide are approaching their design sensitivities and will soon start long data-taking runs. One of these laser interferometers is the GEO 600 detector which was built by a British/German collaboration near Hannover in Germany. The GEO 600 detector is one of the four detectors used within the LIGO scientific collaboration (LSC) to search for gravitational waves. Even though the GEO 600 detector [1] has a shorter baseline than the LIGO detectors [2], advanced technology is used in GEO 600 with the goal of achieving, in a limited bandwidth, a sensitivity close to the sensitivity of the LIGO detectors. Over the next two years the LSC detectors will acquire data in the 'S5 science run.' As soon as the VIRGO interferometer reaches its design sensitivity, the operation of an international network of interferometers will search for gravitational waves. The GEO 600 detector uses the technique of signal recycling which gives the GEO team the flexibility to optimize the detector sensitivity at different frequencies. One possible optimization strategy for GEO 600 in coincidence searches could be to try to match the sensitivity of the LIGO and VIRGO detectors as closely as possible. A second strategy might be to optimize GEO 600 for higher frequencies to complement the sensitivity of LIGO and VIRGO. An analysis of the limiting noise sources of GEO 600 shows that significant improvements can only be made for this high-frequency detection mode, and thus the GEO-HF project will concentrate on upgrades that will reduce noise sources at frequencies in the $\mathrm{kHz}$ region where, for example, normal modes in neutron stars [3] or quasi-normal modes in black holes provide interesting gravitational wave sources.

Upgrades to the long-baseline detectors are planned to improve their sensitivities in the long term. For example, the Advanced LIGO project (AdvLIGO) [4] has recently been approved by the National Science Foundation in the USA. The plan is to increase the injected and circulating power, improve the seismic isolation, change the test masses and their suspensions and apply the signal-recycling technique. A program called AdvVIRGO [5] is in the definition phase with the goal of improving the sensitivity of the VIRGO detector. Both projects expect an improvement by a factor of approximately 10 in sensitivity. The large-scale cryogenic gravitational wave telescope (LCGT) [6] is proposed for construction below ground in the Kamioka mine in Japan. This detector will use cryogenic techniques to reduce the thermal noise and its expected sensitivity is close to the sensitivity of AdvLIGO and AdvVIRGO.

Due to limiting infrastructure and a river which prevents any increase in the arm length, GEO 600 cannot compete with the advanced long-baseline detectors at low frequencies and 
no plans exist to upgrade the GEO 600 detector to match the AdvLIGO and AdvVIRGO broadband sensitivities. Some sequential upgrades of the GEO 600 instrument are, however, foreseen to maintain the ability to provide data for the gravitational wave search until the advanced detectors come online. Especially during the down-time of LIGO and VIRGO, an upgraded GEO 600 detector might prove to be very useful. The development, testing and installation of these upgrades between 2006 and 2010 are named 'The GEO-HF Project' and will be described in detail in the following sections of this paper.

\section{Noise sources limiting the sensitivity of GEO 600}

The dominant noise source of GEO 600 for frequencies below $50 \mathrm{~Hz}$ is seismic noise. For higher frequencies a combination of shot noise and thermal noise will limit the GEO 600 sensitivity. Compared with other interferometric gravitational wave detectors, the shape of the shot noise curve is a distinct feature of GEO 600. The position and transmission of the signal-recycling mirror determine the position of the minimum and the bandwidth of the shot noise curve. Hence GEO 600 has the possibility of matching the shot noise curve to the thermal noise and/or to specific targeted sources. One possible operation mode is to tune the shot noise curve in such a way to achieve thermal-noise limited sensitivity in the several hundred Hertz region. This brings the shape of the noise curve close to those of LIGO and VIRGO and is optimal for operating GEO 600 in a network search mode. Hence one constraint on all GEO-HF upgrades is to avoid degrading the detector performance when it is tuned to low frequencies, in order to always allow the possibility of returning to the (broadband) network operation mode. A different possibility is to tune the signal-recycling resonance to higher frequencies and make the bandwidth smaller, to come close to the thermal noise limited sensitivity in the $\mathrm{kHz}$ region. This mode of operation will be called high-frequency mode and will be subject of the rest of this paper.

Figure 1 shows a comparison of the sensitivities of first generation detectors (represented by the LIGO curve) with the proposed advanced detectors and with the thermal noise limit of GEO-HF (see later sections). The figure clearly shows that the thermal noise limited GEO-HF detector can provide useful data, in the network as well as in the high-frequency operation mode. (Please note that a thermal noise limited sensitivity of GEO-HF can only be achieved in a limited bandwidth which is determined by the signal recycling parameters.)

Three noise sources driven by thermal fluctuations are important in the GEO 600 sensitivity: coating thermal noise [7-11], substrate thermal noise [12] and thermorefractive noise [13]. The first two cause a displacement of the mirror surface whereas the third introduces fluctuations in the index of refraction and is relevant for transmissive optical components. The thermorefractive noise is important in GEO 600 as the optical layout has no Fabry-Perot cavities in the interferometer arms which means that fluctuations in the optical path length caused by a fluctuating index of refraction in the beamsplitter have the same relevance as mirror displacements and are not reduced by the finesse of arm cavities as in all other gravitational wave detectors. The substrate thermal noise plotted in earlier GEO 600 sensitivity curves was calculated by assuming a mechanical quality factor of $Q \approx 5 \times 10^{6}$ for the fused silica mirrors and the beamsplitter. Recent experiments [14] and calculations [15] suggest that this value $Q$ can be more than an order of magnitude higher. For this reason we will neglect the mirror substrate contribution to the thermal noise for the rest of this paper.

In the following sections we will discuss the anticipated improvements in the GEO 600 sensitivity in two groups: the first set will assume that we continue to operate the detector with the current mirrors and are therefore limited by their coating thermal noise. The second set of improvements assumes that the fruits of the extensive research on reducing the coating 


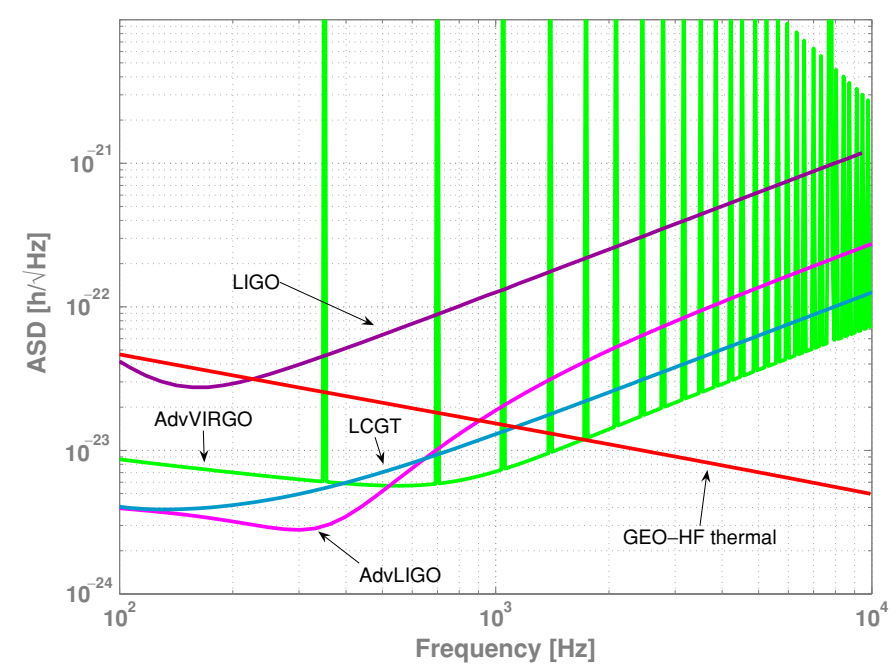

Figure 1. Comparison of the sensitivities of first generation detectors (represented by the LIGO curve) with the proposed advanced detectors and with the thermal noise limit of GEO-HF. The bandwidth and frequency of the shot noise curve of GEO-HF can be tuned to match this thermal noise limit either at low frequencies (for coincidence searches) or at frequencies above $1 \mathrm{kHz}$ to search for high frequency sources.

thermal noise will allow us, in a few years, to acquire new optics with reduced coating thermal noise.

\section{Reducing the sensitivity limit caused by shot noise}

In a signal-recycled interferometer, the optimization of the shot noise limit is a multistage process. An increase of the circulating power in the interferometer will improve the signal to shot-noise ratio. This is true up to the power level at which the radiation pressure noise will introduce mirror displacement noise that is higher than the apparent displacement noise caused by the shot noise on the output photodiode. For all power increments anticipated in the GEO-HF project the radiation pressure contribution can be neglected. (Since we concentrate on the high-frequency region, we will also neglect the additional complication of the optical spring resonance [16] in this paper.) Once the circulating power is maximized the injection of squeezed light into the detector output port could be used to reduce the shot noise at all frequencies (see section 4). After these two broadband improvements are made, the shape of the shot noise curve has to be chosen in such a way as to achieve the best sensitivity for the expected waveform of the gravitational wave source to be explored. Here a trade-off between bandwidth and peak sensitivity has to be made with thermal noise representing an ultimate limit to the maximal peak sensitivity.

Figure 2 shows the modelled noise sources for the GEO 600 detector in its current configuration where the optimal signal-recycling gain is tuned to $1 \mathrm{kHz}$. The first GEO-HF upgrade will be to increase the circulating power by a factor of approximately 10 . The strength of the thermal lens in the beamsplitter will increase, which would lead to a mismatch of the interfering beams and hence to an unacceptably low dark-fringe contrast. In the current GEO 600 configuration, a ring heater [17] is used to compensate for the thermal lens. With a factor of 10 higher power at the beamsplitter a higher level of aberration will be produced and a 


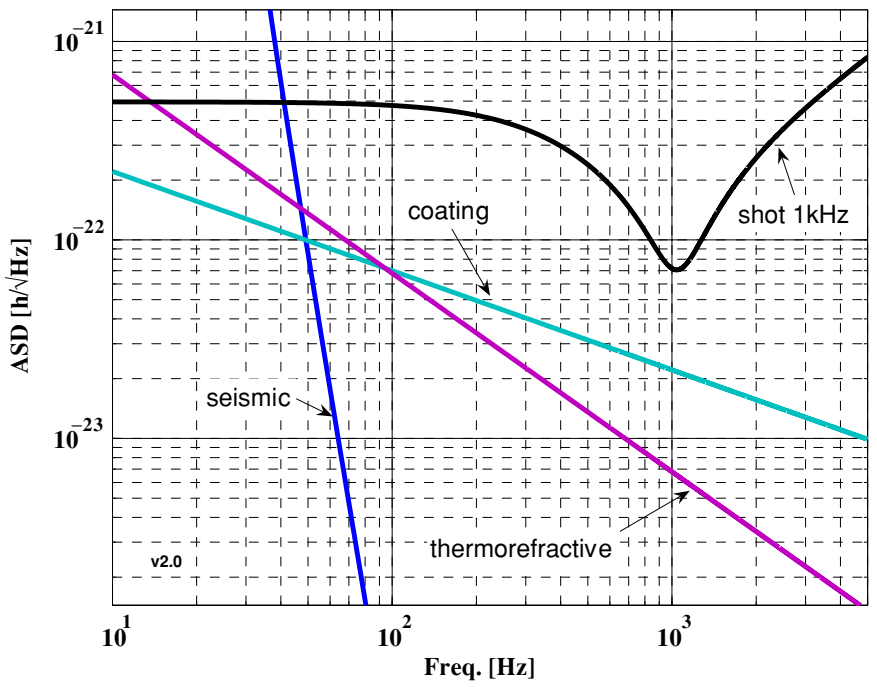

Figure 2. Fundamental noise sources of GEO 600 in the current configuration and for a tuning to $1 \mathrm{kHz}$. For the first upgrades of the GEO-HF project, the thermal noise curves will not change as the main mirrors will not be replaced. Due to a higher circulating power and a change in the signal-recycling bandwidth, we will be able to lower and re-shape the shot noise curve to match the observational goals.

more sophisticated compensation system with a scanning $\mathrm{CO}_{2}$ laser has to be developed and installed.

A laser system with higher output power will be used to increase the circulating power. In a collaboration with the Laser Zentrum Hannover two options are under development both of which use a GEO 600 type $12 \mathrm{~W}$ laser [18] as the front end. The first system with about $50 \mathrm{~W}$ of output power uses four $\mathrm{Nd}$ :vanadate rods in an amplifier configuration. The second system is the laser developed for the AdvLIGO project which uses an injection locking scheme with four Nd:YAG rods in an oscillator configuration to produce $200 \mathrm{~W}$ of output power. The finesse of the two modecleaners will be lowered to avoid problems associated with thermal loading or with instabilities caused by radiation pressure effects during lock acquisition. If the $200 \mathrm{~W}$ source is chosen, the power-recycling factor can be lowered to ease lock acquisition of the dual-recycled interferometer.

With a factor of 10 higher circulating power the shot noise is reduced to the expected level of coating thermal noise at $1 \mathrm{kHz}$. Depending on the detection goal, the signal-recycling bandwidth can be reduced at this point to make the shot noise even smaller. In that case, further sensitivity improvements can only be achieved by a reduction of the coating thermal noise.

\section{Possible improvements related to main optics}

After the identification of coating thermal noise as one of the limiting noise sources for gravitational wave detectors, a significant amount of R\&D has been devoted towards improving the mechanical properties of the dielectric coatings of the mirrors. First measurements indicate that doping of the $\mathrm{Ta}_{2} \mathrm{O}_{5}$ coating material with titania improves the mechanical coating performance [19]. Another idea is to change the layer thickness to reduce the total amount of the lossy coating component [20]. Figure 3 shows the thermal noise contribution for 


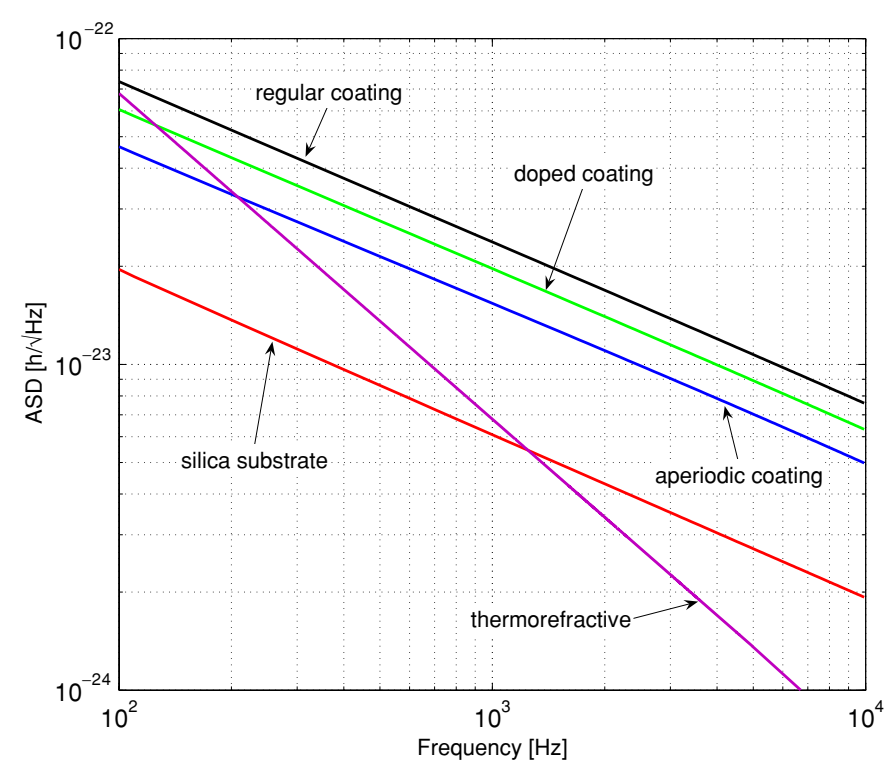

Figure 3. Thermal-noise contributions to the GEO sensitivity. The coating thermal noise is dominating for all frequencies above $100 \mathrm{~Hz}$. Shown are the curves for the expected thermal noise with the currently installed mirrors and two estimates of noise curves improved by $\mathrm{TiO}_{2}$ doping of the $\mathrm{Ta}_{2} \mathrm{O}_{5}$ coating material and by changing the thickness of the different coating layers.

the GEO detector where a high fused-silica quality factor of $2 \times 10^{8}$ was chosen and the best current estimate of the reduced noise with the improved coatings is plotted. The beamsplitter coating is made with fewer layers than the HR coatings and we assume that the coating thermal noise of the beamsplitter is thus less than the main mirror contribution. Hence even opaque mirror materials like silicon could be chosen for a GEO-HF upgrade if future research shows that such materials are advantageous in the coating thermal noise reduction.

For the GEO-HF project, we assume that enough progress on reducing coating thermal noise will be made over the next few years to justify the expense in funds and time of replacing the main mirrors. This will be a large change to the detector as the GEO 600 mirrors are suspended by fused-silica fibres and the installation of a new set of all main mirrors will take several months. The benefit of lower thermal noise would, however, cover the low frequency range as well as the high frequency range and after an optimization of the signalrecycling parameters the GEO 600 sensitivity would improve in the network as well as in the high-frequency detection mode.

A reduction in thermal noise would call for a further improvement of the shot noise which we envisage to be achieved by injecting squeezed light into the output port of the interferometer. This method was first suggested by Caves [21]. It was adopted for the use in GEO by Schnabel et al [22] and recently demonstrated in a table-top experiment [23, 24]. Long optical filter cavities are required to produce squeezing with a frequency-dependent squeezing angle, and the optical components for such a filter cavity will be installed in parallel with the exchange of the main mirrors to reduce detector down times. To make efficient use of the injected squeezed light all mirrors of the filter cavity as well as the main mirrors need to have small optical losses, which sets an additional requirement on the performance of the coatings of the new main mirrors. 


\section{Prototype work}

An important part of the GEO-HF project is the test and preparation of subsystem upgrades and optical configurations in prototypes. These tests are required to keep the down time of the GEO detector to a minimum. One type of test will cover the subsystem interfaces, the installation procedures and the installation tools. Noise measurements in a suspended lownoise environment define a second type of test to be performed on subsystems and on schemes before their installation in GEO 600 - for example the case of a tunable signal-recycling mirror. Subsystems to be developed at the prototype level are a digital control environment, the thermal compensation system, and an improved active isolation system which is needed to reduce rms deviation of the detector from its operation point and to ease lock acquisition. Finally, the prototypes will be used to support the transition of second and third generation technology and configurations from the table-top level towards detector subsystems and realistic interferometer configurations. This means for example testing all reflective optical topologies with gratings as beamsplitters and cavity input mirrors in a suspended interferometer system. Furthermore, investigations that involve radiation pressure effects on suspended mirrors, for example the optical spring effect, can be performed on these prototypes.

\section{Summary}

The GEO 600 detector is expected to reach its design sensitivity in the near future and will be operated in a worldwide network to search for gravitational waves until the second generation detectors like advanced LIGO and advanced VIRGO come online. GEO 600 is the only detector in the network of initial detectors that allows the tuning of its most sensitive detection frequency. This allows the GEO team to operate the detector in a network mode or in a high-frequency mode. The goal of the GEO-HF program is to improve the sensitivity of the GEO detector in the high-frequency mode by reducing the effect of two limiting noise sources, namely shot noise and coating thermal noise. These goals can be achieved by sequential upgrades of the instrument. First the injected laser power will be increased and signal-recycling parameters will be adjusted to reduce the shot noise contribution compared to the coating thermal noise limit. Depending on the availability of mirrors with lower coating thermal noise and low overall optical losses we plan in a second stage to exchange the main mirrors which will allow for efficient use of squeezed light injection into the interferometer output and for a new optimization of the signal-recycling parameters to improve the highfrequency detector performance. In such a configuration, the GEO detector will be the most sensitive detector at high frequencies worldwide and will be used to search for high-frequency sources whenever the detection strategy of the worldwide detector network allows the use of GEO 600 in the high-frequency mode.

\section{Acknowledgments}

The authors would like to thank PPARC in the UK, the BMBF and the state of Lower Saxony in Germany. We also thank M Punturo, G Harry and K Kuroda for providing the sensitivity curves for AdvVIRGO, AdvLIGO and LCGT.

\section{References}

[1] Willke B et al 2002 Class. Quantum Grav. 191377

[2] Sigg D et al 2006 Class. Quantum Grav. 23 S51 
[3] Andersson N and Kokkotas K D Mon. Not. R. Astron. Soc. 2741039

[4] Giaime J et al 2005 Talk presented at 6th Edoardo Amaldi Conference on Gravitational Waves, Okinawa, Japan, 20-24 June 2005

[5] Punturo M et al 2006 J. Phys.: Conf. Ser. 32223

[6] Kuroda K et al 2003 Class. Quantum Grav. 20871

[7] Nakagawa N, Gretarsson A M, Gustafson E K and Fejer M M 2002 Phys. Rev. D 65102001

[8] Harry G M et al 2002 Class. Quantum Grav. 19897

[9] Crooks D R M et al 2002 Class. Quantum Grav. 19883

[10] Penn S D et al 2003 Class. Quantum Grav. 202917

[11] Crooks D R M et al 2004 Class. Quantum Grav. 21 S1059

[12] Liu Y T and Thorne K S 2000 Phys. Rev. D 62122002

[13] Braginsky V B and Vyatchanin S P 2000 Phys. Lett. A 279154

[14] Ageev A et al 2004 Class. Quantum Grav. 213887

[15] Penn S et al available at http://www.ligo.org/pdf_public/penn.pdf

[16] Harms et al 2003 Phys. Rev. D 68 42001-1-8

[17] Willke B et al 2004 Class. Quantum Grav. 21 S417

[18] Zawischa I et al 2002 Class. Quantum Grav. 191775

[19] Harry G LIGO document number P030057-00-D available via http://www.ligo.caltech.edu/dcc/

[20] Pinto I 2005 Private communication

[21] Caves C M 1981 Phys. Rev. D 231693

[22] Schnabel R et al 2004 Class. Quantum Grav. 21 S1054

[23] Vahlbruch H et al 2005 Phys. Rev. Lett. 95211102

[24] Vahlbruch H et al 2006 Class. Quantum Grav. 23 S251 